\title{
ARBEITSETHOS
}

\section{Fehler machen Spaß!}

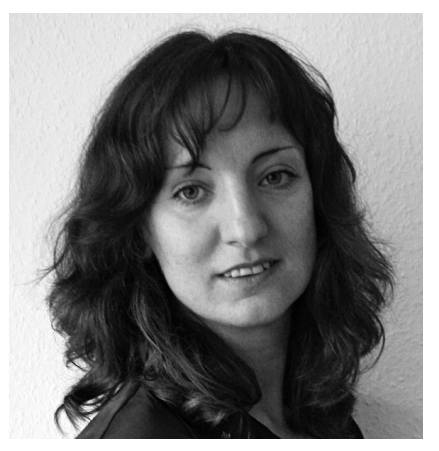

VON SIMONE JANSON

Simone Janson ist Gründerin von Berufebilder.de, laut ZEIT ONLINE eines der meistgelesenen Blogs für Beruf, Bildung und Karriere in Deutschland. Sie ist ist Journalistin und Beraterin, u.a. am Institut für Kommunikation in sozialen Medien (www.ikosom. de), schreibt Lehrwerke für MBAStudiengänge, war Vortragende für die Deutsche Bahn AG oder diverse Hochschulen sowie Koordinatorin für die internationale Konferenz Social Media Week. Der nebenstehende Beitrag basiert auf zwei Artikeln auf dem Blog www.imgriff.de.

redaktion@berufebilder.de

\author{
Damit die Vorteile des Internets und der \\ vernetzten Zusammenarbeit genutzt werden \\ können, ist ein Abschied von den Dogmen des \\ protestantischen Arbeitsethos notwendig. Das \\ digitale Zeitalter fordert neue Fähigkeiten.
}

Es gibt in unserer Gesellschaft ungeschriebene Dogmen: Eines davon ist, dass hundertprozentige Perfektion ein erstrebenswerter Zustand ist. Ein anderes, das Arbeit nur etwas wert ist, wenn sie hart und entbehrungsreich ist; Arbeit muss weh tun. Und schließlich ein anderes Ideal, das eng damit zusammenhängt: Wer Dinge umgehend erledigt, gilt als besonders fleißig - und auch das ist in den Augen unserer Gesellschaft eine äußerst positive Sache.

Das Ideal der protestantischen Arbeitsethik, wie sie unter anderem von dem Soziologen Max Weber beschrieben wurde, ist historisch gesehen eine ziemlich neue Erscheinung. Martin Luther war es, der aus der bis dahin wertneutralen Arbeit eine von Gott auferlegte heilige Pflicht machte und den Müßiggang zur Sünde erklärte. Für die Puritaner wurde es im dann zu einer heiligen Pflicht; materieller Erfolg durch harte Arbeit galt als Gnadenbeweis Gottes.

Möglich wurden diese Veränderungen durch die grundlegende wirtschaftliche Umwälzung jener Zeit: die Industrialisierung. Der rasante Bevölkerungsanstieg führte zu einer verstärkten Nachfrage an Gütern, die bedient werden wollte. Neben dem technischen Fortschritt wurde dazu die optimale Ausnutzung der menschlichen Arbeitskraft durch Zentralisierung der Arbeit in Fabriken und die systematische Aufteilung routinemäßiger Arbeitsabläufe notwendig. Die dafür erforderliche strenge Organisation und die Abhängigkeit von starren Arbeitszeiten wurden ermöglicht durch die Erfindung der Uhr und des elektrischen Lichtes, was erlaubte, auch ohne Tageslicht zu arbeiten.

\section{Digitale Revolution}

Bekanntlich befinden wir uns nun erneut an einem historischen Wendepunkt: Routineaufgaben werden immer mehr von Maschinen übernommen; die Menschen können - und müssen - sich den kreativen Aufgaben zuwenden. Dank Internet, Social Media und mobilen Technologien stehen wir vor einem einschneidenden Paradigmenwechsel in Gesellschaft und Arbeitswelt.

Das Internet bietet beispielsweise hervorragende Chancen der vernetzten Kooperation, in denen Zusammenarbeit auch über große räumliche Entfernungen möglich ist. Social Media bieten wie keine anderen Tools zuvor die Möglichkeit, auch über große Entfernungen in Kontakt zu bleiben. Das ist einer der Gründe für den Erfolg von »Facebook«, was immer man sonst von dem blauen Riesen halten mag. Genau das ist aber die Schwierigkeit: Viele Menschen haben vor allem die Nachteile im Kopf. Denn die Veränderung macht vielen Angst: Ständige Erreichbarkeit und vermeintlicher Dauer-Kommunikationszwang, Teamarbeit bei räumlicher Trennung oder auch ständige Ablenkungen sind Stressoren, die direkt die Leistungsfähigkeit und damit die Produktivität von Unternehmen beeinflussen und denen wir uns stellen müssen.

Oder anders ausgedrückt: Damit wir die Vorteile des Internets und der vernetzten Zusammenarbeit nutzen kön- 
nen, ist der Abschied von den Dogmen des protestantischen Arbeitsethos notwendig: Im digitalisierten Zeitalter sind andere Fähigkeiten gefragt.

den Laden so gut organisiert hat, dass es auch ohne ihn geht, während er sich entspannt und Ideen für die Zukunft ausbrütet. Wohingegen einem Manager,

\section{"Den Spaß an der Arbeit machen sich viele selbst kaputt"}

\section{Aus Fehlern lernen}

Ein Beispiel für die neu geforderten Fähigkeiten im digitalen Zeitalter ist der Umgang mit Fehlern. Leistungen und Produkte müssen nach bisheriger Ansicht perfekt sein. Da jedoch jede Aussage unmittelbar durch das Internet verifizierbar ist, ist das kaum noch möglich. Google geht einen anderen Weg: Neue Dienste werden als unfertige Beta-Versionen zur Verfügung gestellt und durch die Nutzer selbst verbessert. Google steht zu Fehlern, probiert öffentlich im Versuch-und-Irrtum-Verfahren neue Möglichkeiten aus - und entwickelt damit innovative Produkte. Dieses Prinzip könnte das Leitbild einer digitalisierten Unternehmenskultur werden, die fehlgeschlagene Innovationen für Verbesserungen nutzt, statt diese anzuprangern.

Wenn man sich nicht ständig dem Druck aussetzt, etwas Perfektes zu schaffen, dann kann Arbeit sogar Spaß machen. Diese Idee scheint vielen Menschen geradezu ungeheuerlich. Doch Spaß an der Arbeit muss sein, denn nur motivierte Mitarbeiter sind in der Lage, gute Ideen zu entwickeln und Innovationen zu schaffen. Unternehmen wie Google haben das begriffen und bieten ihren Mitarbeitern auch allerlei Freizeitund Sporträume an.

Den Spaß an ihrer Arbeit machen sich viele aber selbst kaputt. Und daran sind sie auch noch teilweise selbst schuld, denn viele glauben, leistungsbereit und fleißig zu sein, wenn sie auch stets erreichbar sind.

Wer hingegen klug ist, steigert mit gelegentlichen Phasen der Unerreichbarkeit sein Ansehen. Denn wenn alle jederzeit erreichbar sind, wird es zum seltenen Luxus, nicht ständig antworten zu müssen.

Einmal längere Zeit nicht erreichbar zu sein, kann zu einem Gütesiegel für eine gute Leitungskraft werden: nämlich, dass er seinen Job versteht, weil er der dauergestresst ständig abrufbereit ist, keine Zeit mehr für seine eigentliche Führungsaufgabe und innovatives Denken bleibt. Der Harvard-BusinessManager empfiehlt als Abschreckungsmaßnahme sogar: "Jedes Mal, wenn ein Manager in dieser Zeit sein Büro anruft, sollte sein Jahresendbonus um 20 Prozent reduziert werden. Jedes Mal wenn ein Mitarbeiter den Manager anrufen muss, würden diesem 10 Prozent seines Bonus abgezogen.«

Sich mal gönnen, Fehler machen und herumprobieren zu dürfen, Spaß haben an der Arbeit und auch immer wieder zur Regeneration abschalten - das alles passt hilft uns, mit Leidenschaft und kreativ bei der Sache zu bleiben und innovative Ideen zu entwickeln.

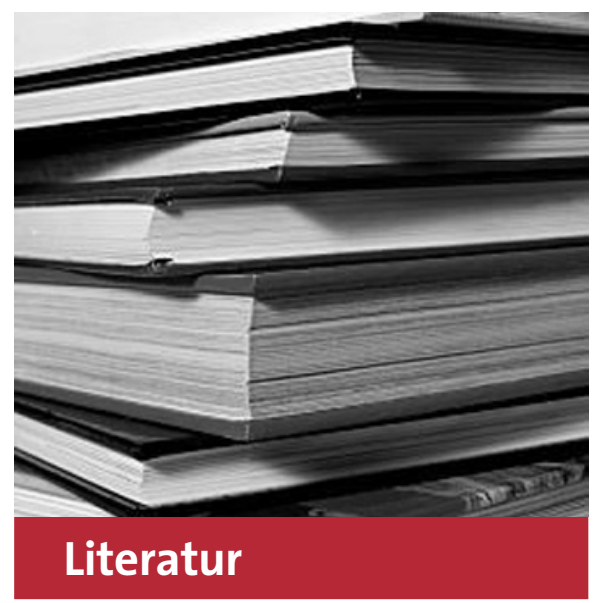

Simone Janson: Nackt im Netz: Wenn Social Media gefährlich wird. Verlag Redline, München 2012. 208 Seiten. 16,99 Euro. ISBN 9783868813135

Simone Janson: Die 110\%-Lüge: Wie Sie mit weniger Perfektion mehr erreichen. Verlag Redline, München 2012. 208 Seiten. 17,99 Euro. ISBN 978-3-86881-363-0.

Simone Janson: Selbstorganisation und Zeitmanagement. Weniger Stress mit strukturiertem Arbeitsablauf. Mehr Motivation durch gute Organisation. Mit Praxistipps und Checklisten. Verlag Redline, München 2007. 192 Seiten. 17,90 Euro. ISBN 978-3-636-01415-3.

\section{Altern in Europa}

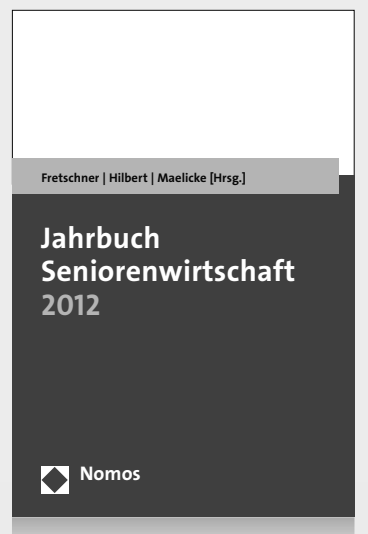

Jahrbuch Seniorenwirtschaft 2012

Herausgegeben von Rainer Fretschner, Josef Hilbert und Bernd Maelicke

2012, 122 S., brosch., 29,- $€$

ISBN 978-3-8487-0037-8

www.nomos-shop.de/20092

Die Europäische Union hat das Jahr 2012 zum Europäischen Jahr für aktives Altern und die Solidarität zwischen den Generationen erklärt. Damit wird einerseits der demographischen Entwicklung in Europa Rechnung getragen und anerkannt, dass die Zukunft Europas nur durch die aktive Gestaltung des demographischen Wandels gewährleistet werden kann. Auf der anderen Seite wird durch die besondere Betonung der Solidarität zwischen den Generationen auf die Notwendigkeit einer gerechten Lastenverteilung zwischen den Generationen aufmerksam gemacht.

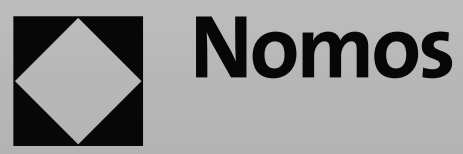

